\title{
ERRATUM
}

Dieter Rehder · João Costa Pessoa

Carlos F.G.C. Geraldes · M. Margarida C.A. Castro

Themistoklis Kabanos · Tamás Kiss · Beate Meier

Giovanni Micera - Lage Pettersson - Maria Rangel

Athanasios Salifoglou - Iztok Turel · Dongren Wang

\section{In vitro study of the insulin-mimetic behaviour of vanadium(IV, V) coordination compounds}

Published online: 24 April 2002

(C) SBIC 2002

\section{J Biol Inorg Chem (2002) 7:384-396}

The correct coordination mode for Ala-His in the peroxovanadium complex $\mathbf{1 5}$ (Fig. 1) is via $\mathrm{N} \epsilon$ of the imidazolyl moiety, as noted in the text and ref. 43. The correct structural formula is shown below.

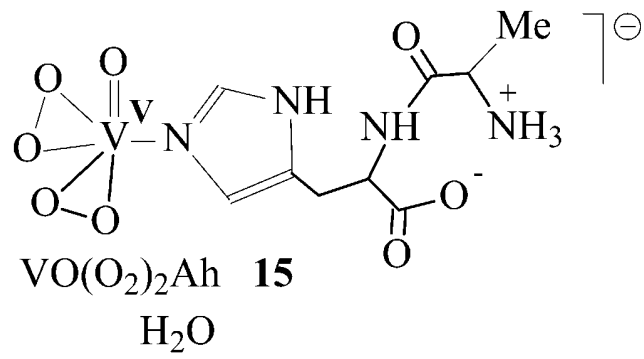

Fig. 1.

B. Meier

85307 Entrischenbrunn 12, Germany

G. Micera

Department of Chemistry,

University of Sassari, 07100 Sassari, Italy

L. Pettersson

Department of Inorganic Chemistry,

University of Umeå, 90187 Umea, Sweden

M. Rangel

CEQUP, Instituto de Ciências Biomédicas de Abel Salazar,

Universidade do Porto, 4099-003 Porto, Portugal

A. Salifoglou

Department of Chemistry,

University of Crete, 71409 Heraklion, Greece

I. Turel

Faculty of Chemistry and Chemical Technology,

University of Ljubljana, 1001 Ljubljana, Slovenia

Department of Chemistry, University of Ioannina,

45100 Ioannina, Greece

T. Kiss

Department of Inorganic and Analytical Chemistry,

University of Szeged, 6701 Szeged, Hungary 